Manuscript is a non-peer reviewed preprint to be submitted to Geophysical Journal International

\title{
Redshift of Earthquakes via Focused Blind Deconvolution of
}

\section{Teleseisms}

\author{
Pawan Bharadwaj, Chunfang Meng, Aimé Fournier, \\ Laurent Demanet, Mike Fehler, \\ Massachusetts Institute of Technology, \\ 77 Massachusetts Ave, Cambridge, MA 02139, USA
}

March 29, 2020

\begin{abstract}
We present a robust factorization of the teleseismic waveforms resulting from an earthquake source into signals that originate from the source and signals that characterize the path effects. The extracted source signals represent the earthquake spectrum, and its variation with azimuth. Unlike most prior work on source extraction, our method is data-driven, and it does not depend on any path-related assumptions e.g., the empirical Green's function. Instead, our formulation involves focused blind deconvolution (FBD), which associates the source characteristics with the similarity among a multitude of recorded signals. We also introduce a new spectral attribute, to be called redshift, which is based on the Fraunhofer approximation. Redshift describes source-spectrum variation, where a decrease in high frequency content occurs at the receiver in the direction opposite to unilateral rupture propagation. Using the redshift, we identified unilateral ruptures during two recent strike-slip earthquakes. The FBD analysis of an earthquake, which originated in the eastern California shear zone, is consistent with observations from local seismological or geodetic instrumentation.
\end{abstract}

\section{Keywords}

Earthquake source observations; Inverse theory; Interferometry; Time series analysis 


\section{Introduction}

Geophysicists perform dynamic rupture simulations on an assumed fault surface to gain insight into the slip distribution and associated rupture evolution of an earthquake. The dynamic and kinematic rupture parameters that control these simulations are mostly unknown; therefore they have to be estimated from geodetic and seismological measurements. In order to estimate them, it would be desirable to directly measure the source pulses at the seismometers and subsequently infer quantities that are informative about the rupture parameters. However, the signals measured in place of those pulses are affected by the subsurface properties through which they propagate before reaching these stations. Thus, instead of measuring the earthquake source signal, each seismic station measures a signal that is a spatio-temporal convolution between the earthquake signal (which is unknown, and of primary interest) and the Green's function of the subsurface (which is also unknown, but of secondary or negligible interest). The Green's function evaluated at a particular station depends on the subsurface characteristics e.g., its structure and intrinsic attenuation, which are also unknown. For the foregoing reasons, an accurate characterization of the earthquake rupture involves a factorization i.e., separation of the ground motion data into the information that originates from the source and information related to the path effects.

This paper considers factorization of primarily the first arriving surfaces waves, termed as R1 (Rayleigh) and G1 (Love $\left.{ }^{1}\right)$ waves, contained in the long-period records of intermediate-magnitude strike-slip earthquakes. It has to be noted that the source pulses will become dispersed as their frequency components travel at different phase velocities along the Earth's surface. As a result, the factorization cannot rely on the identification or windowing of individual phases in the seismograms. Moreover, owing to the uncertainties in the path effects i.e., the phase velocities, such a factorization amounts to so-called "blind deconvolution", where both factors, the Green's function and source signals are unknown. To our knowledge, this paper presents the first demonstration of the required factorization, thanks to a recent advance in deconvolution methodology, namely "focused blind deconvolution" (FBD, introduced by Bharadwaj et al., 2019). Our factorization provides complementary information on the rupture characteristics compared to existing methods that rely on isolating the P-wave (pressure-wave) arrivals (e.g, Tocheport et al., 2007). Warren and Shearer (2006) estimated the source spectra and the rupture directivity by stacking the windowed the P-wave arrivals from globally distributed earthquakes.

The factorization of the seismograms is challenging and generally not solvable, because of the unknown trade-off between the source $s$ and path effects $g$ i.e., extracting one requires assumptions about the other. However, FBD compares a multitude of records (e.g., Plourde and Bostock, 2017) due to the same source, and

\footnotetext{
1 "G" after Beno Gutenberg.
} 
identifies the similarities among them through a formal analysis. Subsequently, it associates the similarities to the spectrum of $s$, and the dissimilarities to $g$. For the success of FBD, we require that the receivers span a wide range of azimuth-angles and distances with respect to the rupture. In recent years, large numbers of seismometers have been deployed, so this requirement can easily be satisfied.

\section{$1.1 \quad$ Pervious works}

Source estimation is the first step towards earthquake-rupture characterization, and a series of deconvolution results have appeared in the literature that relied on different assumptions. For example, Ulrych (1971), Ulrych et al. (1972), and Clayton and Wiggins (1976) introduced homomorphic deconvolution to seismology, so that the deconvolution problem was reduced to a linear-filtering operation in the cepstral domain.

\subsubsection{Empirical Green's function}

In contrast to FBD, a collection of existing source estimation techniques rely heavily on a reconstruction of the convolution operator i.e., the Green's function. Numerous source-estimation methods e.g., the wellknown SCARDEC (seismic source characteristics retrieved from deconvolving, Vallée et al., 2011; Vallée and Douet, 2016) method, construct the deconvolution operator via synthetic wave modeling (Kikuchi and Kanamori, 1982, 1986, 1991; Lay et al., 2009). Synthetic modeling of the P phases is possible, as it doesn't involve any complex path effects, except for intrinsic attenuation of waves; Appendix C presents P-phase source estimation comparing both FBD and SCARDEC algorithms. However, reliable construction of surface waves is difficult owing to the uncertainties in the subsurface parameters that are necessary for the wave modeling. Another class of source-estimation techniques that are widely used today utilizes the records from a weaker earthquake in the fault region to construct the so called 'Empirical Green's Function' (EGF, Hartzell, 1978; Lanza et al., 1999; Vallée, 2004; McGuire, 2004). The assumption here is that the weaker earthquake occurs due to a rupture over smaller characteristic fault length, and therefore is impulsive. Depending on the fault region under consideration, there may not be any suitable weaker earthquake, of reasonable signalto-noise ratio, available as an EGF. Furthermore, an automatic processing of a large number of earthquakes is difficult with the EGF approach as it involves a careful selection of the deconvolution operator from the record database. Moreover, recently Wu et al. (2019) identified significant source complexity of a weak, moment magnitude scale $\mathrm{Mw} \approx 4.0$ earthquake. Plourde and Bostock (2017) also recognized that no event is sufficiently weaker for use in the construction of an EGF, and proposes a simultaneous multichannel deconvolution of two different collocated earthquakes, whose records are assumed to share common path effects. 
Many case-studies report a successful source estimaton using the EGF approach, when a suitable weaker earthquake is available. Ammon et al. (1993) used regional and teleseismic surface waves, and a suitable EGF, to analyze the rupture directivity of the 1992 Landers earthquake. López-Comino et al. (2012) used a $\mathrm{Mw} \approx 4.6$ foreshock and a $\mathrm{Mw} \approx 3.9$ aftershock to construct the EGF and observed a clear directivity effect of the 2011 Lorca earthquake (Spain).

\subsubsection{Teleseismic backprojection}

Another method that is widely used to study evolution of the ruptures is backpropagation or time-reversal (Larmat et al., 2006; Meng et al., 2016; Yin and Denolle, 2019) of teleseismic P waves. Unlike the EGF methods, they only assume the kinematics between different locations on the fault to the receivers, in order to perform time-reversal. Similar to FBD, the advantage is that this method is able to exploit the coherency among waveforms recorded at multiple seismic stations. However, this method not only suffers from the uncertainties in the ray paths, but also fails to utilize multiple recorded phases. As a result, the existing source estimation techniques are limited to utilizing the $\mathrm{P}$-wave arrivals in the seismograms, which can be backpropagated if given the correct kinematics.

\subsubsection{Rupture estimation from near-source stations}

A collection of methods (Heaton and Helmberger, 1977; Olson and Apsel, 1982) use finite-fault modeling and dense near-source stations to directly infer the rupture parameters. Somala et al. (2018) discuss an adjoint-state formulation for least-squares fitting of the near-source ground motion to optimize for the source parameters related to the finite-fault modeling. Similarly, Gallovic et al. (2019) developed a Bayesian framework to estimate the uncertainties during the determination of these parameters. Their experimental results show that the errors in the assumed velocity model can severely impact the source inversion results. FBD utilizes only the regional and global stations, as opposed to the near-source stations in these methods. Note that this will allow FBD to analyze earthquakes with a sparse distribution or even absence of near-source stations.

\section{Redshift in an Earthquake Spectrum}

Our primary goal is the robust estimation of the earthquake source spectrum using the aforementioned factorization of the teleseismic waveforms. In this section, we first assume a kinematic source model for a fault that is vertical. Then, we associate the parameters of this source model to the features e.g., redshift or Doppler shift in the estimated source spectrum. 

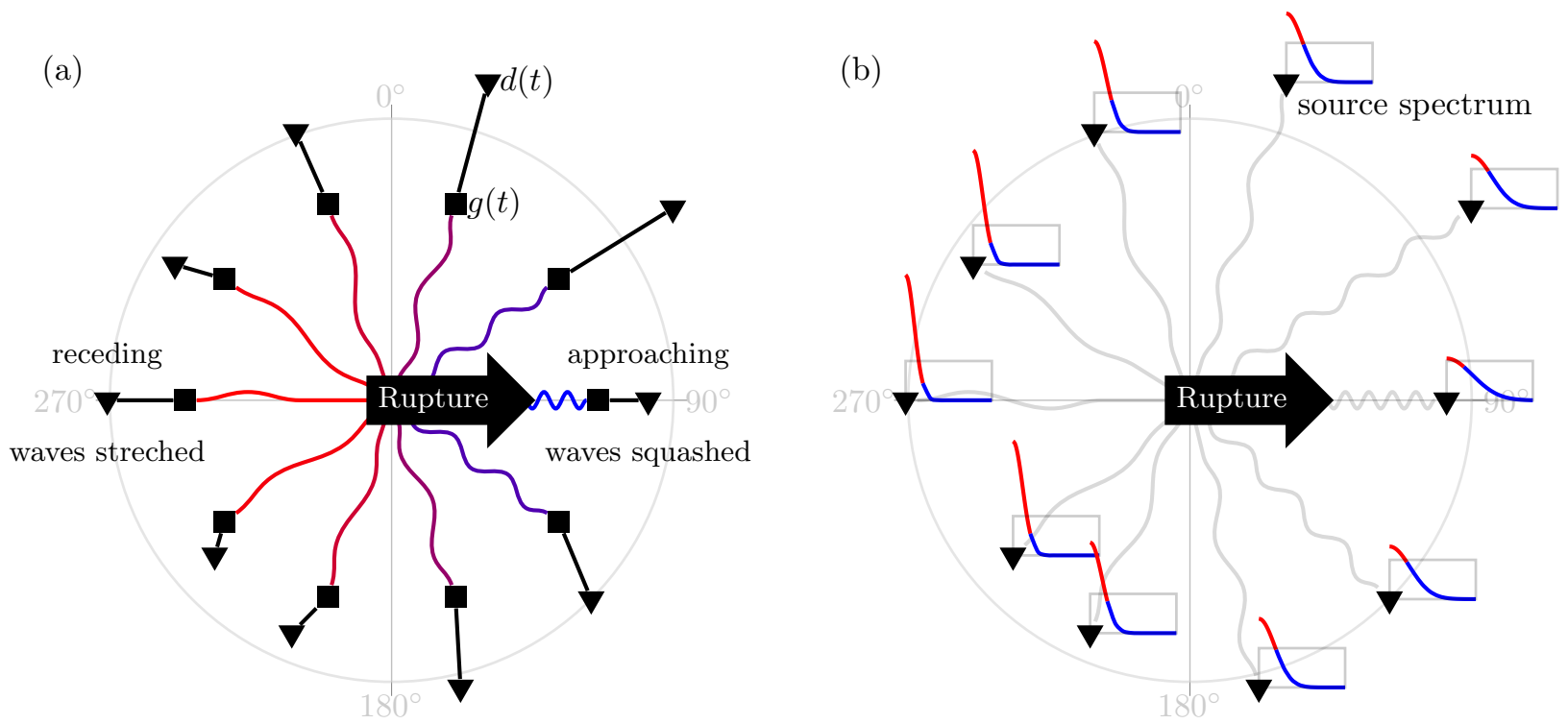

Figure 1: Schematic of waves emitted due to a rupture propagating from west (azimuth $\theta=270^{\circ}$ ) to east $\left(90^{\circ}\right)$. a) Blue waves emitted towards the east are shortened, while the red waves traveling towards the west are lengthened. These waves undergo complex scattering (squares) before they reach the receivers (triangles), resulting in a challenging source-spectrum estimation problem. b) FBD factorizes the measurements, effectively removes the complex scattering or path effects and directly estimates the source spectra (red-blue graphs) at the receivers. The variability of the normalized source spectrum with $\theta$ can be used to infer the kinematic rupture parameters.

We set a cylindrical coordinate system with origin $O$, radius $r$, azimuth $\theta$, and height $z$. The fault plane extends from $r=0$ to $L$ along the radial line $\theta=90^{\circ}$ (i.e., from $x=0$ to $L$ along $y=0$ ), and from $z=0$ to $H \ll L$ along the cylindrical axis. A unidirectional rupture starts at the hypocenter, located at $O$, and propagates along the radial line. The kinematic rupture model, explained in Appendix A, is simplified using the Fraunhofer approximation to represents the waves recorded at $(r, \theta)$ on the surface $z=0$ as

$$
d(t ; r, \theta) \approx s(\cdot ; \theta) *_{t} g(\cdot ; r, \theta) .
$$

Here, the path effects, for a given moment tensor, are denoted by a convolution operation (eq. A.7) in time with a function $g(t ; r, \theta)$, which corresponds to the response due to impulsive force couples acting at the hypocenter. The apparent source pulse emitted in the direction of azimuth $\theta$ is given by the function:

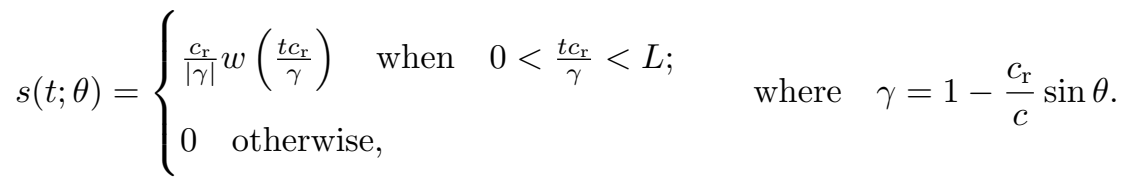

In the above equation, $\gamma$ roughly varies between 0 and 2 , owing to the common observation that rupture speed $c_{\mathrm{r}}$ is comparable to wave speed $c$. The function $w$ depends on $H$ and represents the distribution of 
stress drop along the radial line of the fault. (Note that we have substituted $\psi=\theta-90^{\circ}$ in eq. A.5 - this substitution only being valid for the waves that depart from the fault along radial lines - so in section 4.3 , we primarily analyze the surface waves emitted from steeply-dipping faults.)

The source model in eq. 2 is less restrictive compared to a model that regards the fault as a stationary point source i.e., it also incorporates the seismic wavelength $\lambda$ that is comparable to $L$. However, as in eq. A.4, it requires that the receivers are located at large distances $r \gg 2 L^{2} / \lambda$. Accordingly, in section 4.3 , we analyze the above-mentioned surface waves in the long-period seismograms:

- recorded at teleseismic distances with $r>1600 \mathrm{~km}$ i.e., epicentral distance greater than $15^{\circ}$;

- that contain dominant frequencies less than $0.1 \mathrm{~Hz}$ - as a result $\lambda \gtrsim 40 \mathrm{~km}$;

- from intermediate-magnitude $(6.0<\mathrm{Mw}<6.5)$ earthquakes typically with $L \approx 60 \mathrm{~km}$.

In eq. 2 , it can be noted that the source-function $w$ argument is scaled depending on

1. the speed $c_{\mathrm{r}}$ of the rupture propagation;

2. the direction $\theta$ relative to the rupture propagation;

3. and the speed $c$ of the propagating waves in the source region.

Therefore, if the rupture is approaching a station $\left(\theta=90^{\circ} \Rightarrow|\gamma| \ll 1\right)$ then the source function $w$ is shortened as depicted in Fig. 1a. Accordingly, as a result of the scaling property of the Fourier transform, its amplitude spectrum is lengthened over the frequency $\omega$, as shown in the Fig. 1b. On the other hand, if the rupture is receding $\left(\theta=270^{\circ} \Rightarrow \gamma=1+c_{\mathrm{r}} / c \approx 2\right)$ from a station then the source function $w$ is lengthened in time, resulting in a shortened-frequency amplitude spectrum of the source. This causes an apparent shift in the corner frequency (Brune, 1970; Savage, 1972), which is considered in the Haskell fault model (Madariaga, 2015). Ben-Menahem (1961) studied the quotient of the spectral amplitudes, called the directivity function, of waves leaving the rupture in opposite directions.

Unfortunately, the time-scaled source pulse i.e., the apparent source pulse $s$ is affected through convolution by the properties of the subsurface that the signal propagates through before reaching these stations. Such effects prevent us from directly observing the apparent source pulse at the stations. In the following sections, we will present a factorization of the records $d$ of an earthquake to eliminate the path effects, as depicted in Fig. 1. 


\section{Focused Blind Deconvolution}

FBD requires that multiple receivers span a wide range of azimuth angles $\theta$ and distances $r$ relative to the rupture. For such a set of receivers, a temporal-index window $t \in\left\{T_{1}, \ldots, T_{2}\right\}$, relative to the origin time of the earthquake, has to be applied in order to roughly isolate either the $\mathrm{P}$ or $\mathrm{S}$ (shear-wave) phases. Depending on the temporal window, FBD outputs either the $\mathrm{P}$ - or the $\mathrm{S}$-wave source spectrum as a function of $\theta$, denoted with $|\hat{S}|$. For example, when the starting time $T_{1}=0$ and the ending time $T_{2}$ is roughly chosen to be the mean of PPP (twice-reflected P) and SS (once-reflected S) arrival-times, the windowed records will mostly contain P-wave energy and $|\hat{S}|$ corresponds to the P-wave source spectrum. Otherwise, the least-squares misfit in FBD is dominated by the high-amplitude surface-wave phases, resulting in the estimation of S-wave source spectrum. Even though the surface phases are primarily analyzed in the rest of the paper, the FBD of $\mathrm{P}$ phases is straightforward, as discussed in Appendix C. Note that the difference between the estimated P and S source spectra can be used to further characterize the ruptures; we leave such an investigation to a later study. We consider many azimuthal bins $\Theta \subsetneq\left[0^{\circ}, 360^{\circ}\right)$, each with $n$ receivers,

such that the variability of each restriction $\left.|\hat{S}|\right|_{\theta \in \Theta}$ can be ignored. Therefore, we have $s(t ; \theta) \approx s(t) \forall \theta \in \Theta$, resulting in a single-input multiple-output model

$$
d_{i}(t)=s *_{t} g_{i} .
$$

Here, the subscript $i \in\{1,2, \ldots, n\}$ denotes an index of a receiver that records the ground motion $d_{i}(t)$, or a spatial location where the Green's function $g(\cdot, t)$ is evaluated as $g_{i}(t)$. We denote a vector of records by $\left[d_{i}\right]:\left\{T_{1}, \ldots, T_{2}\right\} \rightarrow \mathbb{R}^{n}$, and a vector of Green's functions by $\left[g_{i}\right]:\left\{T_{1}, \ldots, T_{2}\right\} \rightarrow \mathbb{R}^{n}$. The duration length of each element of $\left[d_{i}\right]$ and $\left[g_{i}\right]$ is therefore $T_{2}-T_{1}+1$, which was chosen to be long enough that each $d_{i}$ can contain an identical source pulse $s:\{0, \ldots, T\} \rightarrow \mathbb{R}$. It is important to note that the FBD results are insensitive to the choice of the duration length $T+1$ of $s$-provided the length is long enough to capture the source effects.

In every $\Theta$, the intention is to blindly factorize i.e., deconvolve the ground motion $\left[d_{i}\right]$ in eq. 3 into the path effects $\left[g_{i}\right]$ and the source $s$, with much fewer and simpler assumptions about these factors, compared to those made in conventional methods. A suitable algorithmic approach, related to multichannel blind deconvolution (BD), is a least-squares fit of $\left[d_{i}\right]$ to jointly optimize two unknown functions $\left[g_{i}\right]$ and $s$. The joint optimization can be suitably carried out using alternating minimization (Ayers and Dainty, 1988; Sroubek and Milanfar, 2012): in one cycle, we fix one function and optimize the other, and then fix the other and optimize the first. Several cycles are expected to be performed to reach convergence. However, it is well 
known that $\mathrm{BD}$ is not solvable, due to non-uniqueness, without making assumptions on at least one of the two unknown factors. These assumptions determine the admissible trade-off between $\left[g_{i}\right]$ and $s$ during the optimization.

Accordingly, we employ focused blind deconvolution (FBD), which first reduces the trade-off in BD by considering a least-squares fitting of interferometric or cross-correlated records, instead of the raw records. And second, it determines all the remaining trade-off (except for an overall uniform phase) by associating the dissimilarities among the multiple records to $\left[g_{i}\right]$, while attributing similarities to $s$. Our examples below demonstrate that these associations are valid as long as the receivers are placed at dissimilar locations i.e., their separation distances are much larger than the wavelength.

One import aspect of FBD is the following reformulation that is simpler to solve, due to the reduced trade-off, as it only estimates the unknown source auto-correlation and interferometric path effects.

Definition 1 (IBD: Interferometric Blind Deconvolution). The interferometric record between $i$ th and $j$ th receivers is given by

$$
d_{i j}(t)=\left\{d_{i} \otimes d_{j}\right\}(t)=\{\underbrace{s \otimes s}_{s_{\mathrm{a}}}\} *_{t}\{\underbrace{g_{i} \otimes g_{j}}_{g_{i j}}\}
$$

where $\{u \otimes v\}(t)=\bar{u} *_{t} v$ defines temporal cross-correlation and $\bar{u}$ temporally reverses $u$. IBD aims for a least-squares fitting of an $(n+1) n / 2$-vector, denoted by $\left[d_{11}, d_{12}, \ldots, d_{1 n}, d_{22}, d_{23}, \ldots, d_{2 n}, \ldots, d_{n n}\right]$ or simply $\left[d_{i j}\right]$, of the unique interferometric records between every possible receiver pair:

$$
\left(\hat{s}_{\mathrm{a}},\left[\hat{g}_{i j}\right]\right)=\underset{s_{\mathrm{a}},\left[g_{i j}\right]}{\arg \min } \sum_{k=1}^{n} \sum_{l=k}^{n} \sum_{t=T_{1}-T_{2}}^{T_{2}-T_{1}}\left\{d_{k l}(t)-\left\{s_{\mathrm{a}} * g_{k l}\right\}(t)\right\}^{2} .
$$

Along the similar lines of BD, it jointly optimizes two functions, namely the interferometric Green's function $\left[g_{i j}\right]:\left\{T_{1}-T_{2}, \ldots, T_{2}-T_{1}\right\} \rightarrow \mathbb{R}^{(n+1) n / 2}$ and the auto-correlated source function $s_{\mathrm{a}}:\{-T, \ldots, T\} \rightarrow \mathbb{R}$

The motivation behind dealing with $\left[d_{i j}\right]$ is that the cross-correlation operation discards the phase information from the Fourier representation of the source. Therefore, the admissible trade-off between the path effects $\left[g_{i j}\right]$ and the source $s_{\mathrm{a}}$ is reduced, compared to trade-off between $\left[g_{i}\right]$ and $s$ in BD. The remaining trade-off, pertaining to the amplitude spectrum of the source, is determined in FBD by regularizing with a focusing functional:

$$
J=\sum_{k=1}^{n} \sum_{t=T_{1}-T_{2}}^{T_{2}-T_{1}} t^{2} g_{k k}(t)^{2}
$$


FBD minimizes $J$ i.e., the energy of the auto-correlated Green's functions $g_{i i}$ multiplied by the lag time, to result in a solution where the $g_{i}$ are heuristically as white (in the frequency domain) as possible. As shown by Bharadwaj et al. (2019), simultaneously maximizing the whiteness of any $g_{i}$ promotes its dissimilarity from all the $g_{j \neq i}$. Therefore, for the success of FBD, it is important that the true $g_{i}$ are sufficiently dissimilar. For instance, in the limit that the true $g_{i}$ are all equal to each other, FBD just outputs the temporal Kronecker $\delta(t)$ for the $g_{i}$, making the $s$ equal the $d_{i}$. In our experiments, we ensure that the "sufficiently dissimilar" requirement is satisfied by choosing receivers separated by distances $r$ all much larger than the wavelength. Note that, for a given receiver configuration, the width $|\Theta|$ of each azimuthal bin $\Theta$ determines the range of $r$; we choose each $|\Theta|$ sufficiently large such that receivers span a wide range of $r$, while small enough to provide some azimuthal resolution.

Now, after estimating source auto-correlation in every $\Theta$, the next step is to normalize them such that $\left.\hat{s}_{\mathrm{a}}(0)\right|_{\theta \in \Theta}=1$. Then, the Fourier representation of $\hat{s}_{\mathrm{a}}$ can be used to construct the normalized source spectrum. For every $\Theta$, the duration of the apparent source time function is given by the time necessary for the envelope $\mathrm{E}\left(\left.\hat{s}_{\mathrm{a}}\right|_{\theta \in \Theta}\right)$ to decrease below a chosen threshold. The envelope operator $\mathrm{E}$ computes the absolute value of the analytic representation of a real-valued signal. The final trivial step is to combine the outputs together over all $\Theta$ to form the estimated source properties over the entire interval of $\theta$. If the azimuthal distribution of the receivers is non-uniform, then the results have a variable azimuthal resolution that we smooth using a spline interpolation.

\subsection{Why Maximally White?}

To illustrate the importance of the focusing constraint, we use random signals to represent hypothetical path effects at $n=20$ receivers. The amplitude spectra $\left|G_{i}\right|$ are plotted in red in Fig. $2 \mathrm{~b}$. It can be noticed that the spectra are dissimilar to each other, suggesting a sufficiently dissimilar (hypothetical) receiver configuration. These spectra are now multiplied in the frequency domain with an arbitrary source spectrum to produce measurements corresponding to the blue-colored spectra of Figure 2. We then solve the IBD problem (eq. 4), without using the focusing constraint, to factorize the recorded spectra into the corresponding source and path-effect spectra. The estimated spectra $\left|\hat{G}_{i}\right|$ and $|\hat{S}|$ are presented in Fig. 2a. Even though we obtain a low least-squares misfit at convergence i.e., $\left|D_{i}\right|=|\hat{S}|\left|\hat{G}_{i}\right| \forall i$, the $\left|\hat{G}_{i}\right|$ don't match with the true spectra plotted in Figure 2b. More importantly, it is physically unreasonable that the $\left|\hat{G}_{i}\right|$ are similar to each other, provided that the receivers have a dissimilar configuration to begin with. The similarity in this case is indicated by the common notch at the frequency indicated by the dashed line in Figure 2a. On the other 
a) Undesired Factorization (without focusing constraint)

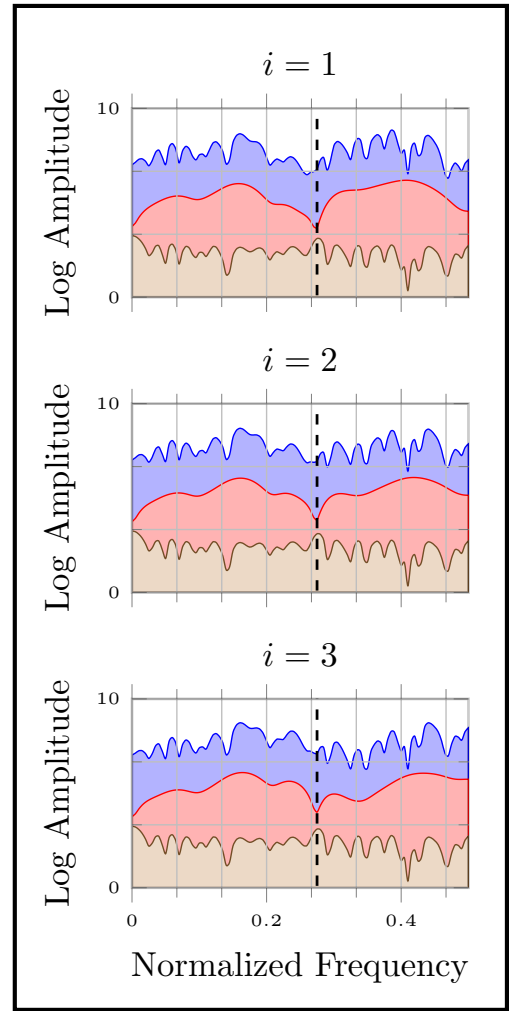

b) Desired Factorization (with focusing constraint)

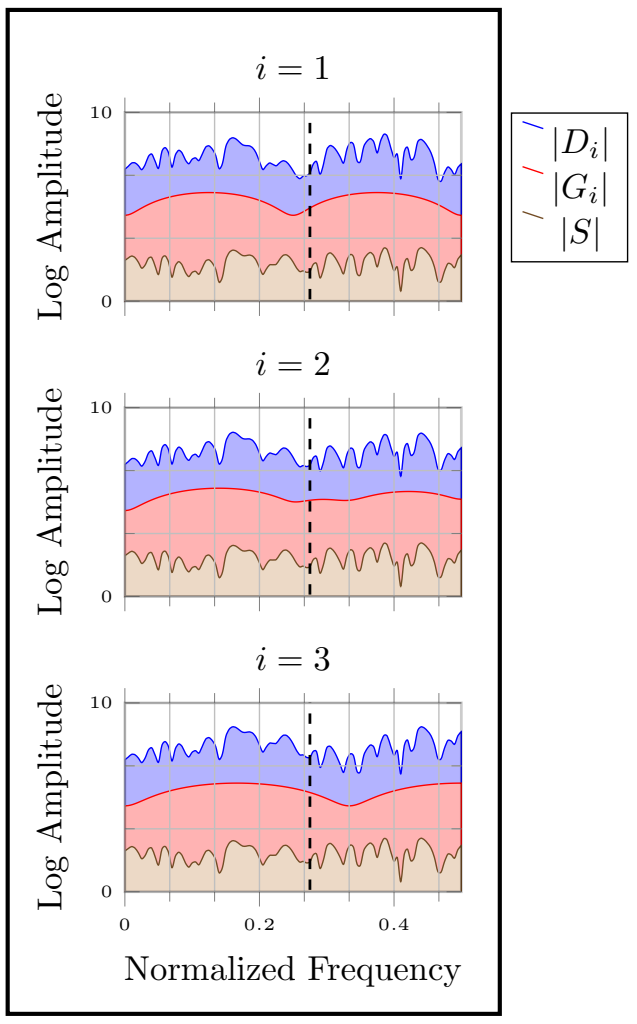

Figure 2: Two possible factorizations of the recorded spectra $\left|D_{i}\right|$, associated with the solutions of the IBD problem in eq. 4 . In each of the plots the recorded spectrum is satisfied i.e., $\log \left|D_{i}\right|=\log \left|\hat{G}_{i}\right|+\log |\hat{S}|$ as implied by eq. 3 . In factorization (a), the estimated spectra associated with the path effects $\left|\hat{G}_{i}\right|$ are similar to each other for different $i$ e.g., they have a common notch at the frequency indicated by the dashed line. Therefore, factorization (a) is physically unreasonable, provided that the receivers are separated by distances much larger than the wavelength. The focusing constraint in FBD obtains the factorization (b) that exactly matches the true factors - note that the $\left|\hat{G}_{i}\right|$ in this factorization are not only more white but also more dissimilar to each other.

hand, the focusing constraint $J$ is designed to choose a solution, where $\left|G_{i}\right|$ are maximally white. Which means, the solutions similar to this that have common notches will be avoided by the focusing constraint, therefore promoting dissimilarity among the $\left|G_{i}\right|$. In this experiment, FBD converges to the true solution (Figure $2 \mathrm{~b}$ ), leading us to conclude that seeking maximally white $\left|G_{i}\right|$ is equivalent to seeking maximally dissimilar $\left|G_{i}\right|$. Therefore, in the framework of FBD, the similarities in the recorded spectra are extracted and identified as source $|\hat{S}|$ effects, leaving path effects $|\hat{G}|$ to be dissimilar.

\section{Applications}

For a given earthquake, FBD estimates the apparent source auto-correlation $\hat{s}_{\mathrm{a}}(t ; \theta)$, and its zero-phase Fourier representation i.e., the apparent power spectrum $|\hat{S}(\omega ; \theta)|^{2}$ at angular frequency $\omega$. The benefits of 
this methodology include:

1. at any given azimuth $\theta$, the time duration of the apparent source pulse can be determined using that of $\hat{s}_{\mathrm{a}}$;

2. $|\hat{S}(\omega ; \theta)|$ can be inspected for spectral attributes associated with source characteristics e.g., how unilateral is the rupture;

3. more generally, $|\hat{S}(\omega ; \theta)|$ can be used as input to finite-fault inversion to directly infer the rupture parameters, without being affected by the uncertainties in the subsurface models;

4. assuming that multiple earthquakes share identical path effects, the variation of $|\hat{S}(\omega ; \theta)|$ among these earthquakes provides an accurate relative magnitude of each earthquake.

Now, we demonstrate the first two benefits, while leaving the others for future research.

\subsection{Redshift Attribute}

Redshift is a spectral attribute of a rupture propagating almost unilaterally. It is related to the frequencyscaling of the source spectrum as discussed in section 2 (eq. 2). For a given earthquake spectrum and a choice of two different frequency bands, red and blue for low- and high-frequency bands respectively, we:

1. compute the spectral energy of $|\hat{S}|$ in the bands as a function of the azimuth-bin $\Theta$, resulting in a spectral-energy vs azimuth plot;

2. and inspect if the energy in the red band is dominant in a particular direction, corresponding to a dominant blue energy in the opposite direction.

Characteristic 2 of the source-spectrum variation is referred to as redshift. Inspecting the FBD estimated (normalized) source spectra $|\hat{S}(\omega ; \theta)|$ for redshift will help us identify unilateral ruptures from those that are more complex. Note that, as a consequence of the normalization $\hat{s}_{\mathrm{a}}(0 ; \theta)=1$, the sum of spectral energy over frequency should be a constant for each $\theta$. In this work, we have arbitrarily chosen the low- and highfrequency bands for the analysis. Ideally, the redshift attribute should be quantified using a more robust measure e.g., the wide-band ambiguity function (Weiss, 1994; Sibul and Ziomek, 1981), which we leave for future research.

\subsection{Synthetic Experiment}

We now present a 2-D numerical experiment that demonstrates the benefits of FBD for rupture characterization. We record both the horizontal- and vertical-component displacement due to a rupture propagating 
unilaterally along $\theta=90^{\circ}$. As depicted in Fig. 3b, 100 receivers surround the source and span a range of distances $r$ from 15 to $32 \mathrm{~km}$. The waves are modeled using an elastic finite-element solver (Meng and Wang, 2018) in a homogeneous spatial domain with both $x$ and $y$ from -32 to $32 \mathrm{~km}$. We didn't add any noise to the synthetic wavefield in this experiment, as FBD has already been tested in the presence of Gaussian white noise by Bharadwaj et al. (2019). We deliberately set reflective, instead of absorbing, boundary conditions to create complex path-specific effects due to multiple scattering. Note that comparably complex path effects could also result from a heterogeneous velocity structures; again, we refer the reader to Bharadwaj et al. (2019) for synthetic experiments involving complex velocity structures. Moreover, this 2-D experiment only involves the scattered $\mathrm{P}$ and $\mathrm{S}$ waves, but similar experiments can also be performed using surface waves, which are considered later in the next subsection.

We employ FBD to estimate $\hat{s}_{\mathrm{a}}(t ; \theta)$ from the full-wavefield records - the envelope of $\hat{s}_{\mathrm{a}}$ (color) and its duration (dashed curve) are plotted in Fig. 3a with lag time $t>0$ on the radial axis and $\theta$ on the azimuthal axis. We isolated the first-arriving S-wave pulses from the records, using a rectangular time window $\Pi$, at $90^{\circ}$ and $270^{\circ}$ to obtain $\Pi d\left(t ; r, 90^{\circ}\right)$ and $\Pi d\left(t ; r, 270^{\circ}\right)$ respectively. These pulses at a particular distance $r$ are plotted in Figs. 3d and 3e in both temporal and Fourier domains. Using eq. 1, we write $\Pi d(t ; r, \theta) \approx g_{\mathrm{S}}(\cdot ; r, \theta) *_{t} s(\cdot ; \theta)$, where $g_{\mathrm{S}}$ denotes the direct (i.e., no scattering) S-wave component of the Green's function. As the function $g_{\mathrm{S}}$ is invariant to $\theta$ for the homogeneous velocity structure under consideration, the difference between the durations of $s\left(t ; 90^{\circ}\right)$ and $s\left(t ; 270^{\circ}\right)$ should be equal to that of $\Pi d\left(t ; r, 90^{\circ}\right)$ and $\Pi d\left(t ; r, 270^{\circ}\right)$. In Fig. 3d-e, observe that the difference of $\approx 1$ s in S-pulse durations, as depicted by the envelopes of the auto-correlated pulses, is consistent with FBD-estimated duration-difference between $\hat{s}_{\mathrm{a}}\left(t ; 90^{\circ}\right)$ and $\hat{s}_{\mathrm{a}}\left(t ; 270^{\circ}\right)$ (Fig. 3a). Also, we plotted the normalized spectra of the S-wave pulses in these plots to observe that the pulse at $270^{\circ}$ has dominant low frequencies compared to that at $90^{\circ}$. This attribute is consistent with FBD-estimated spectral energy vs azimuth plot in Fig. 3c. In this plot, as the sum of spectral energy over frequency is constant for each $\theta$, the radial axis gives the percentage of total energy in a given band.

\subsection{Application to Recorded Earthquakes}

We now use FBD in the source-spectrum analysis of two earthquakes with magnitude $\mathrm{Mw} \leq 6.5$. In recent years, a large number of seismometers have been deployed, which facilitate the capture of the source pulse at a wide range of azimuths $\theta$ and distances $r$, making FBD application feasible. With regard to the source model discussed in the previous sections, we only consider strike-slip earthquakes that ruptured almostvertical faults at shallow depths of $\approx 15 \mathrm{~km}$. The earthquake locations and moment-tensor solutions, listed 
(a)

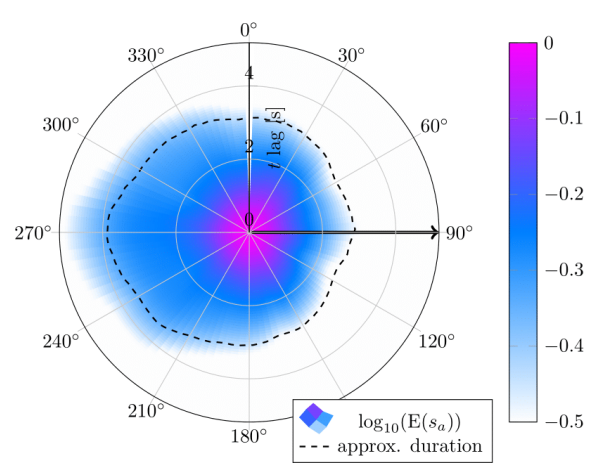

(d) S-wave to the west $\left(\theta=270^{\circ}\right)$

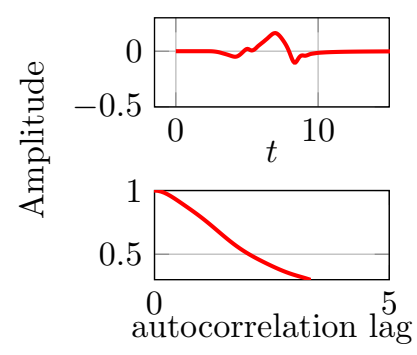

(b) Vertical-displacement snapshot

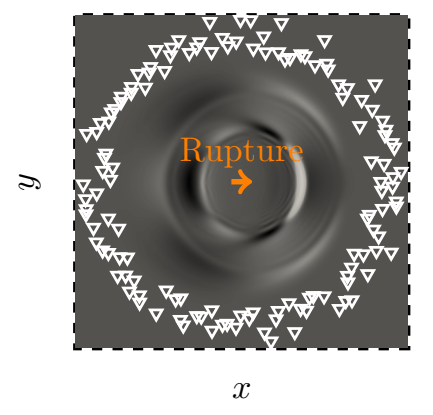

(c)

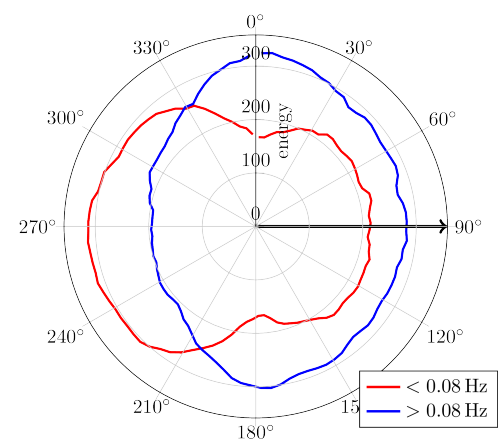

Figure 3: A synthetic experiment. a) The envelope $\mathrm{E}\left(\hat{s}_{\mathrm{a}}(t ; \theta)\right)$ of the FBD-estimated auto-correlated source pulse is plotted (color) as a function of the lag time (radius) and azimuth. The dashed curve indicates the source-pulse duration after smoothing along $\theta$. b) Vertical displacement due to a rupture, colored in gray-scale as a function of the horizontal $x$ and vertical $y$ spatial coordinates, before the $\mathrm{P}$ and $\mathrm{S}$ waves are scattered by the boundaries (dashed lines) of the medium. Only receiver positions with $r>25$ are marked by white triangles in order not to obscure the wavefield. c) The energy of the FBD-estimated source spectrum, in both the low (red) and high (blue) frequency bands, is plotted to depict the redshift. In order to validate the FBD results, the direct S-wave pulse on the opposite sides of the rupture (see text) is plotted in (d) and (e), respectively. The dashed vertical line separates the two frequency bands of (c). 
(a) Nicobar Islands, India

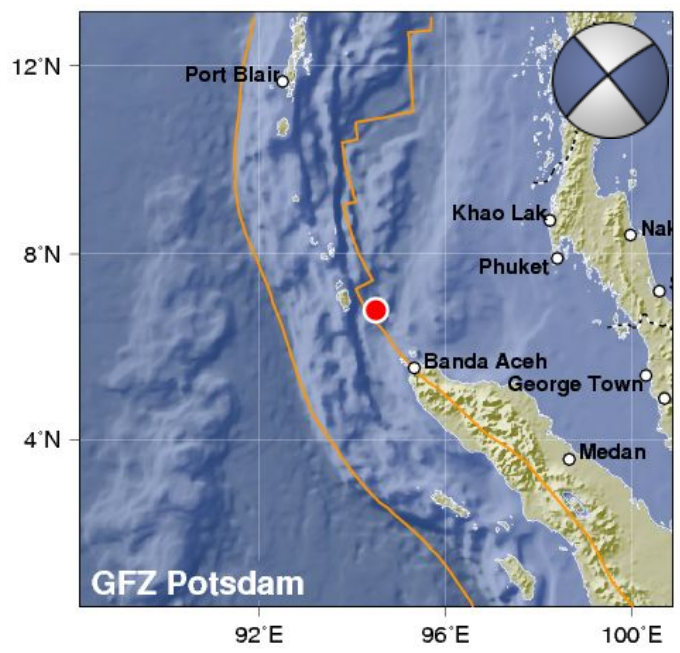

(b) California, USA

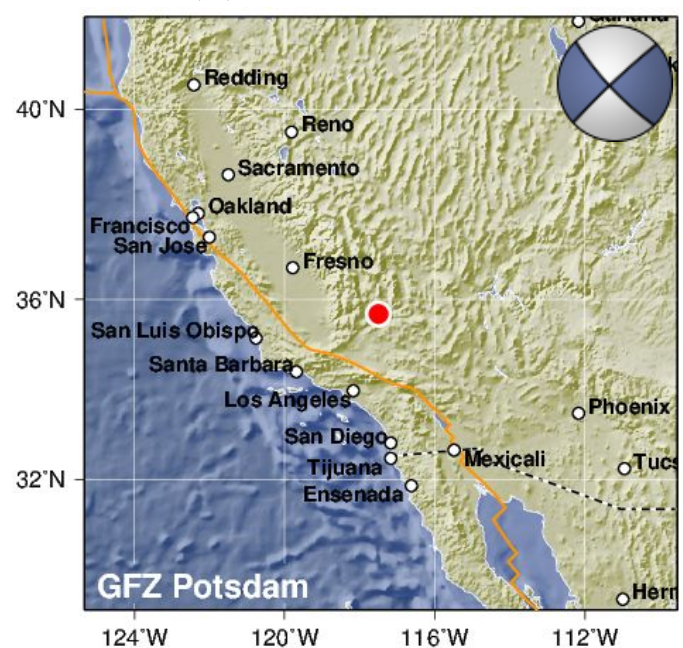

Figure 4: Two strike-slip earthquakes that are analyzed in this paper. Major faults in the region are delineated by orange curves, and moment tensor solutions are inserted. Courtesy: GEOFON Program Hanka and Kind (1994), GFZ Potsdam.

in Table 1, are plotted in Figs. 4 and 5.

Table 1: List of earthquakes along with two possible moment-tensor solutions. Courtesy: GEOFON Earthquake Information Service (Geoforschungsnetz, Hanka and Kind, 1994).

\begin{tabular}{lcccccc}
\hline Name, Date, Mw & Latitude & Longitude & Depth & Strike $\left(^{\circ}\right)$ & Dip $\left(^{\circ}\right)$ & Rake $\left(^{\circ}\right)$ \\
\hline Nicobar, 2015-11-08, 6.5 & $6.79^{\circ} \mathrm{N}$ & $94.50^{\circ} \mathrm{E}$ & $15 \mathrm{~km}$ & 321,230 & 87,82 & $171,-2$ \\
California, 2019-07-04, 6.4 & $35.69^{\circ} \mathrm{N}$ & $117.46^{\circ} \mathrm{W}$ & $14 \mathrm{~km}$ & 137,227 & 82,87 & 177,8
\end{tabular}

For each earthquake, we have downloaded long-period records, with $1 \mathrm{~Hz}$ sampling rate, from 20 supported international data centers (see Data and Resources). Only stations with epicentral distance greater than $15^{\circ}$ were selected, as plotted in the Figs. 5a and 5d. At each seismic station, we utilize multiple components of the recorded displacement, which primarily contain the first-arriving surface waves known as R1 (Rayleigh) and G1 (Love) waves, which are the largest-amplitude arrivals.

The pre-processing of the records is relatively simple. We first window the records with a boxcar function of a duration $\approx 6750$ s following the origin time. Each record is then standardized to have zero mean and unit variance with respect to time, and the inverse of its energy before the P-wave arrival is used as a proxy for signal-to-noise-ratio (SNR). Then, we remove the noisy records with SNR below a certain threshold. Finally, we perform an important step i.e., instrument correction, without which we notice that the instrument response contaminates the FBD-extracted similarity among the records. Again, note that we associate the similarity among the records with the source effects; therefore, it is important that there is no artificial similarity in the recorded spectra due the instrument response of the seismometers. The pre-processed 
records are input to $\mathrm{FBD}$ as $d(t ; r, \theta)$.

\subsubsection{Nicobar (08 November 2015) $\mathrm{Mw}=6.5$}

This strike-slip earthquake ruptured a known fault in a region SE of the Andaman Island (see 4). The teleseismic stations that were utilized in the FBD analysis are plotted in Fig. 5a. The estimated apparent source pulse auto-correlation $\hat{s}_{\mathrm{a}}$, plotted in Fig. $5 \mathrm{~b}$, indicates that the source duration is $\approx 15 \mathrm{~s}$ longer in the NW compared to the SE direction. In the spectral energy vs azimuth plot, the spectral energy is computed in three different frequency bands, where the seismometers have high instrument responses, as plotted in Fig. 5c. These results, similar to those in Figs. 3b and 3c, indicate a unilateral rupture propagation, along the SE trend. Accordingly in Fig. 6, the source spectrum exhibits frequency scaling, with higher corner frequency in the direction of the rupture propagation, and vice versa. The rupture propagation is consistent with one of the two possible strike directions indicated by the moment tensor in Fig. 5a.

\subsubsection{California (04 July 2019) $\mathrm{Mw}=6.4$}

This is a foreshock of the Mw $=7.1$ July 5 mainshock in the 2019 Ridgecrest sequence that occurred as the result of shallow strike slip faulting in the crust of the North America plate. The FBD analysis of the July 5 mainshock (USGS, 2019b) is presented in Appendix B. Compared to the mainshock, the FBD-extracted spectrum for this earthquake indicates a relatively simple rupturing, with dominant propagation towards SW. That is: 1) a shorter source duration is noticed in the SW direction relative to NE, as shown by the $\hat{s}_{\text {a }}$ plot in Fig. 5e; 2) the stations in the SE direction record dominant high frequencies - as is evident from the spectral-energy vs azimuth plot in Fig. 5f. These results are consistent with the direct observations, which suggest that the event ruptured a previously unnoticed NE-SW trending fault. Moreover, the aftershocks following this particular event also aligned along the NE-SW trend (USGS, 2019a). Again, note that the rupture propagation is along one of the two possible strike directions, as indicated by the moment tensor in Fig. 5d.

\section{Conclusions}

We have demonstrated that focused blind deconvolution (FBD) is a powerful data-driven tool for factorizing teleseismic records into source and path effects. Instead of relying on source- or path-related assumptions e.g., the empirical Green's function, FBD characterizes an earthquake source by associating it with the similarity among a multitude of records. However, there is a potential problem with this method: it may not succeed due to a number of simplifications (like azimuth-binning and the Fraunhofer approximation) that 
(a)

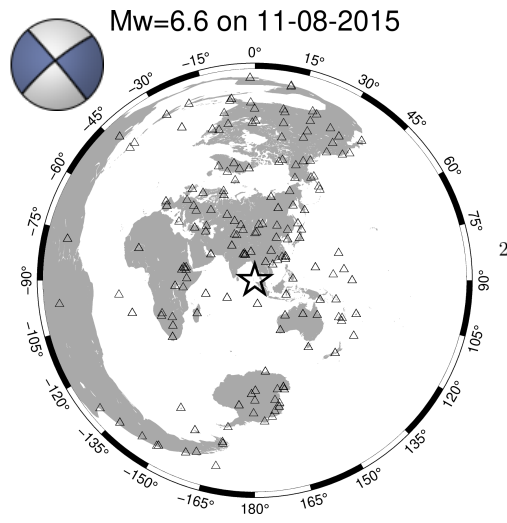

(d)

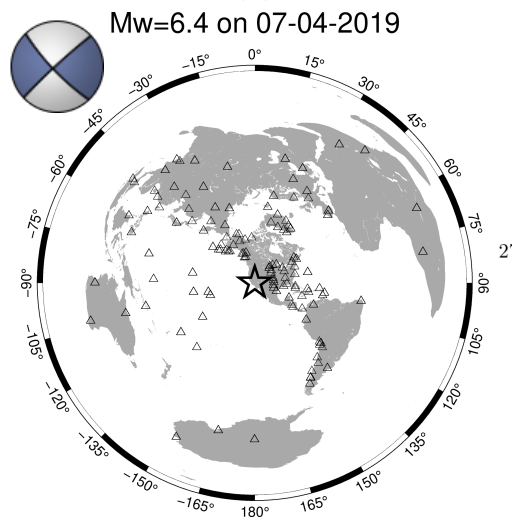

(b)

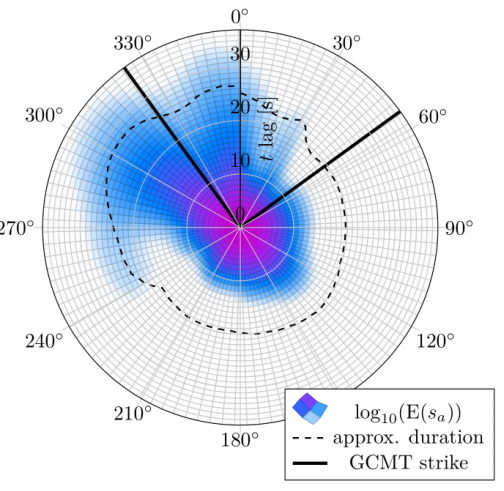

(e)

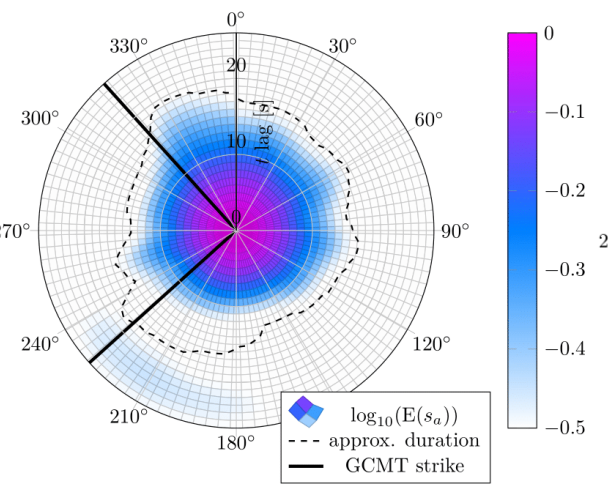

(c)
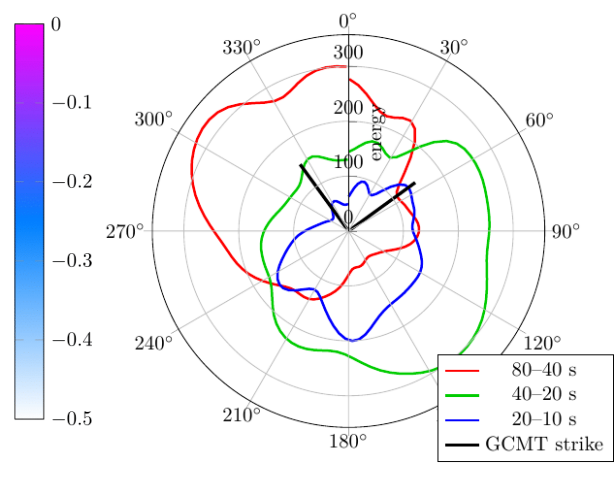

(f)

Figure 5: FBD of two recorded strike-slip earthquakes. a) and d): event (star) and station (triangles) locations. GEOFON moment tensor solutions are inserted. b) and e): the envelope of the estimated autocorrelated apparent source pulse, $\mathrm{E}\left(\hat{s}_{\mathrm{a}}(t ; \theta)\right)$ is plotted along with the two possible strike directions (solid radial lines) — note the variation of the source time duration with azimuth. c) and f): the source spectral energy vs azimuth plot in three different frequency bands indicates redshift. Labels indicate ranges of period $2 \pi / \omega$ in seconds. 


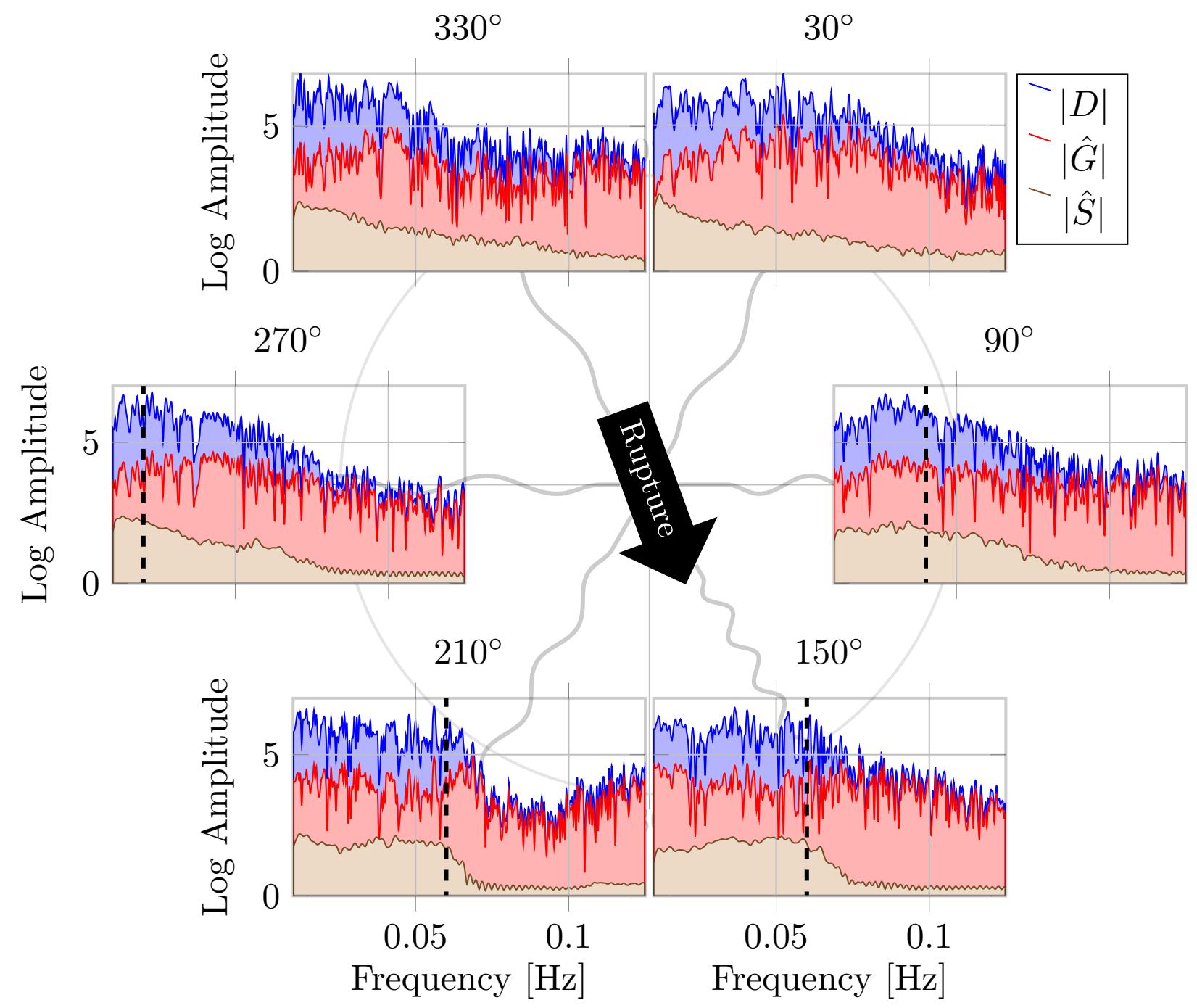

Figure 6: FBD factorizes the recorded spectra $|D|$, due to the Nicobar earthquake, into the source $|\hat{S}|$ and the path $|\hat{G}|$ at multiple azimuths. Note that the source spectrum exhibits frequency scaling, with higher corner frequency (dashed line) in the direction of the rupture propagation (indicated by an arrow) and vice versa. 
were made to arrive at the convolutional model, and there is no theoretical guarantee that FBD performs a physically meaningful factorization even for the convolutional model.

In our numerical experiments, FBD extracted the earthquake source spectra from the surface waves of intermediate-magnitude, shallow strike-slip earthquakes. These spectra are complementary to the ones extracted from other methods using isolated P-wave arrivals. They were further analyzed to identify unilaterally-propagating ruptures during the earthquakes; a potential extension is to robustly estimate the rupture velocity. The FBD results of one of the recent recorded earthquakes that originated in Ridgecrest, California, are consistent with observations from local seismological and geodetic instrumentation - this showcases the potential of FBD to analyze earthquakes without the need of local instrumentation.

\section{Data and Resources}

We are grateful to the organizations who provide and manage data: Global Seismographic Network (GSN) is a cooperative scientific facility operated jointly by the Incorporated Research Institutions for Seismology (IRIS), the United States Geological Survey (USGS), and the National Science Foundation (NSF), under Cooperative Agreement EAR-1261681. The earthquake records from 20 supported international data centers were downloaded using obspyDMT (Hosseini and Sigloch, 2017). The pre-processing of the records is performed using ObsPY toolbox (Beyreuther et al., 2010). Earthquake information was obtained from the GEOFON programme (Hanka and Kind, 1994) of the GFZ (Geoforschungszentrum) German Research Centre for Geosciences using data from the GEVN (GEOFON Extended Virtual Network). The Global Centroid Moment Tensor Project database (www.globalcmt.org/CMTsearch.html, Dziewonski et al., 1981) was also searched for such information. Some plots were made using the Generic Mapping Tools (www.soest.hawaii.edu/gmt, Wessel et al., 2013).

\section{Acknowledgments}

We thank our colleagues Nafi Toksöz, Matt Li, Aurelien Mordret, Tom Herring, Hongjian Fang and Michael Floyd for providing insight and expertise that greatly assisted this research. We thank Martin Mai from King Abdullah University of Science and Technology for his informative commentary of a draft version. The material is based upon work assisted by a grant from Equinor. Any opinions, findings, and conclusions or recommendations expressed in this material are those of the authors and do not necessarily reflect the views of Equinor. LD is also supported by AFOSR grant FA9550-17-1-0316. 
351

352

\section{References}

Aki, K. and P. G. Richards

2002. Quantitative Seismology.

Ammon, C. J., A. A. Velasco, and T. Lay

1993. Rapid estimation of rupture directivity: application to the 1992 landers $(\mathrm{ms}=7.4)$ and cape mendocino $(\mathrm{ms}=7.2)$, california earthquakes. Geophysical research letters, 20(2):97-100.

Ayers, G. and J. C. Dainty

1988. Iterative blind deconvolution method and its applications. Optics letters, 13(7):547-549.

Ben-Menahem, A.

1961. Radiation of seismic surface-waves from finite moving sources. Bulletin of the Seismological Society of America, 51(3):401-435.

Beyreuther, M., R. Barsch, L. Krischer, T. Megies, Y. Behr, and J. Wassermann

2010. ObsPy: A Python Toolbox for Seismology. Seismological Research Letters, 81(3):530-533.

Bharadwaj, P., L. Demanet, and A. Fournier

2019. Focused Blind Deconvolution. IEEE Transactions on Signal Processing, 67(12):3168-3180.

Brune, J. N.

1970. Tectonic stress and the spectra of seismic shear waves from earthquakes. Journal of geophysical research, 75(26):4997-5009.

Clayton, R. W. and R. A. Wiggins

1976. Source shape estimation and deconvolution of teleseismic bodywaves. Geophysical Journal International, 47(1):151-177.

Dziewonski, A. M., T.-A. Chou, and J. H. Woodhouse

1981. Determination of earthquake source parameters from waveform data for studies of global and regional seismicity. Journal of Geophysical Research: Solid Earth, 86(B4):2825-2852.

Gallovic, F., L. Valentova, J.-P. Ampuero, and A.-A. Gabriel 2019. Bayesian Dynamic Finite-Fault Inversion: 1. Method and Synthetic Test. Preprint, EarthArXiv.

Hanka, W. and R. Kind

1994. The GEOFON Program. Annals of Geophysics, 37(5). 
Hartzell, S. H.

1978. Earthquake aftershocks as Green's functions. Geophysical Research Letters, 5(1):1-4.

Heaton, T. H. and D. V. Helmberger

1977. A study of the strong ground motion of the Borrego Mountain, California, earthquake. Bulletin of the Seismological Society of America, 67(2):315-330.

Hosseini, K. and K. Sigloch

2017. ObspyDMT: A Python toolbox for retrieving and processing large seismological data sets. Solid Earth, 8(5):1047-1070.

Kikuchi, M. and H. Kanamori

1982. Inversion of complex body waves. Bulletin of the Seismological Society of America, 72(2):491-506.

Kikuchi, M. and H. Kanamori

1986. Inversion of complex body waves-II. Physics of the earth and planetary interiors, 43(3):205-222.

Kikuchi, M. and H. Kanamori

1991. Inversion of complex body waves-III. Bulletin of the Seismological Society of America, 81(6):23352350.

Lanza, V., D. Spallarossa, M. Cattaneo, D. Bindi, and P. Augliera

1999. Source parameters of small events using constrained deconvolution with empirical Green's functions. Geophysical Journal International, 137(3):651-662.

Larmat, C., J.-P. Montagner, M. Fink, Y. Capdeville, A. Tourin, and E. Clévédé

2006. Time-reversal imaging of seismic sources and application to the great Sumatra earthquake. Geophysical Research Letters, 33(19).

Lay, T., H. Kanamori, C. J. Ammon, A. R. Hutko, K. Furlong, and L. Rivera 2009. The 2006-2007 Kuril Islands great earthquake sequence: THE 2006-2007 KURIL ISLANDS EARTHQUAKES. Journal of Geophysical Research: Solid Earth, 114(B11).

López-Comino, J.-Á., F. d. L. Mancilla, J. Morales, and D. Stich

2012. Rupture directivity of the 2011, mw 5.2 lorca earthquake (spain). Geophysical Research Letters, $39(3)$.

Madariaga, R.

2015. Seismic Source Theory. In Treatise on Geophysics, Pp. 51-71. Elsevier. 
McGuire, J. J.

2004. Estimating Finite Source Properties of Small Earthquake Ruptures. Bulletin of the Seismological Society of America, 94(2):377-393.

Meng, C. and H. Wang

2018. A finite element and finite difference mixed approach for modeling fault rupture and ground motion. Computers \& Geosciences, 113:54-69.

Meng, L., A. Zhang, and Y. Yagi

2016. Improving back projection imaging with a novel physics-based aftershock calibration approach: A case study of the 2015 Gorkha earthquake. Geophysical Research Letters, 43(2):628-636.

Olson, A. H. and R. J. Apsel

1982. Finite faults and inverse theory with applications to the 1979 Imperial Valley earthquake. Bulletin of the Seismological Society of America, 72(6A):1969-2001.

Plourde, A. P. and M. G. Bostock

2017. Multichannel Deconvolution for Earthquake Apparent Source Time Functions. Bulletin of the Seismological Society of America, P. ssabull;0120170015v1.

Savage, J.

1972. Relation of corner frequency to fault dimensions. Journal of geophysical research, 77(20):3788-3795.

Sibul, L. and L. Ziomek 1981. Generalized wideband crossambiguity function. In ICASSP '81. IEEE International Conference on Acoustics, Speech, and Signal Processing, volume 6, Pp. 1239-1242.

Somala, S. N., J.-P. Ampuero, and N. Lapusta

2018. Finite-fault source inversion using adjoint methods in 3-D heterogeneous media. Geophysical Journal International, 214(1):402-420.

Sroubek, F. and P. Milanfar

2012. Robust multichannel blind deconvolution via fast alternating minimization. IEEE Transactions on Image Processing, 21(4):1687-1700.

Stein, E. M. and G. Weiss 2016. Introduction to Fourier Analysis on Euclidean Spaces (PMS-32). Princeton University Press. 
Tocheport, A., L. Rivera, and S. Chevrot

2007. A systematic study of source time functions and moment tensors of intermediate and deep earthquakes. Journal of Geophysical Research: Solid Earth, 112(B7).

Ulrych, T. J.

1971. Application of homomorphic deconvolution to seismology. GEOPHYSICS, 36(4):650-660.

Ulrych, T. J., O. G. Jensen, R. M. Ellis, and P. G. Somerville

1972. Homomorphic deconvolution of some teleseismic events. Bulletin of the Seismological Society of America, 62(5):1269-1281.

\section{USGS}

2019a. Ridgecrest M 6.4; $11 \mathrm{~km} \quad$ SW of Searles Valley, CA. https://earthquake.usgs.gov/earthquakes/eventpage/ci38443183/executive.

\section{USGS}

2019b. $\quad$ Ridgecrest M $\quad$ M.1; $\quad 11 \mathrm{~km} \quad$ SW of Searles Valley, CA. https://earthquake.usgs.gov/earthquakes/eventpage/ci38457511/executive.

Vallée, M.

2004. Stabilizing the Empirical Green Function Analysis: Development of the Projected Landweber Method. Bulletin of the Seismological Society of America, 94(2):394-409.

Vallée, M., J. Charléty, A. M. Ferreira, B. Delouis, and J. Vergoz

2011. SCARDEC: A new technique for the rapid determination of seismic moment magnitude, focal mechanism and source time functions for large earthquakes using body-wave deconvolution. Geophysical Journal International, 184(1):338-358.

Vallée, M. and V. Douet

2016. A new database of source time functions (STFs) extracted from the SCARDEC method. Physics of the Earth and Planetary Interiors, 257:149-157.

Warren, L. M. and P. M. Shearer

2006. Systematic determination of earthquake rupture directivity and fault planes from analysis of longperiod p-wave spectra. Geophysical Journal International, 164(1):46-62.

Weiss, L. G.

1994. Wavelets and wideband correlation processing. IEEE Signal Processing Magazine, 11(1):13-32. 
Wessel, P., W. H. F. Smith, R. Scharroo, J. Luis, and F. Wobbe

2013. Generic Mapping Tools: Improved Version Released. Eos, Transactions American Geophysical Union, 94(45):409-410.

Wu, Q., X. Chen, and R. E. Abercrombie

2019. Source Complexity of the 2015 Mw 4.0 Guthrie, Oklahoma Earthquake. Geophysical Research Letters, 46(9):4674-4684.

Yin, J. and M. A. Denolle

2019. Relating teleseismic backprojection images to earthquake kinematics. Geophysical Journal International, 217(2):729-747.

\section{Appendix A Fraunhofer's Approximation}

An active fault surface causing an earthquake can be regarded as a surface distribution of body forces (Aki and Richards, 2002). The kinematic dislocation model (Madariaga, 2015) assumes that these equivalent body forces are activated in a sequence, depending on the parameter(s) that determine the propagation of the slip. We consider a unidirectional rupture propagation along the length $L$ of a fault plane $\Xi$. The fault plane is assumed to be a rectangle that has a small height $H \ll L$. We denote an infinitesimal surface element at $\boldsymbol{\xi}=\left(\xi_{1}, \xi_{2}\right)$ on the fault by $\mathrm{d} \Xi$, where $\xi_{1}$ and $\xi_{2}$ are local two-dimensional coordinates in the length- and height-directions, respectively. In three dimensions, the $i$ th component of the far-field displacement at $(\mathbf{x}, t)$ due to a displacement discontinuity across a surface element at $\boldsymbol{\xi}$ can be approximated as:

$$
u^{i}(\mathbf{x}, t ; \boldsymbol{\xi}) \approx \sum_{j, k=1}^{3} \int \mathcal{G}^{i j, k}(\mathbf{x}, t-\tau ; \boldsymbol{\xi}) m^{j k}(\tau ; \boldsymbol{\xi}) \mathrm{d} \tau
$$

where $m^{j k}$ denotes the $(j, k)$ th component of the moment density tensor and $\mathcal{G}^{i j, k}$ denotes the $k$ th spatial derivative of the $(i, j)$ th component of the elastodynamic Green's tensor. We now assume an instantaneous slip such that the dependency of the moment density tensor on the time $\tau$ can be ignored. We also assume that the components of the moment density tensor do not vary relative to each other resulting in $m^{j k}(\boldsymbol{\xi})=$ $h(\boldsymbol{\xi}) m^{j k}\left(\boldsymbol{\xi}_{0}\right)$, where $h(\boldsymbol{\xi})$ is proportional to the stress drop at $\boldsymbol{\xi}$ and $\boldsymbol{\xi}_{0}=(0,0)$ is the hypocenter. Rewriting eq. A.1 with these assumptions results in:

$$
u^{i}(\mathbf{x}, t ; \boldsymbol{\xi}) \approx h(\boldsymbol{\xi}) g^{i}(\mathbf{x}, t ; \boldsymbol{\xi}), \quad \text { where } \quad g^{i}(\mathbf{x}, t ; \boldsymbol{\xi})=\sum_{j, k} \mathcal{G}^{i j, k}(\mathbf{x}, t ; \boldsymbol{\xi}) m^{j k}\left(\boldsymbol{\xi}_{0}\right)
$$


In this paper, we refer to the terms 'Green's function' and 'path effects' with $g^{i}$ of the above equation, even though it already includes some directivity effects e.g., due to a force couple. Also, note that we have dropped the component $i$ (not to be confused with receiver-label $i$ ) because FBD handles all the measured displacement components identically. Now, consider a constant speed $c_{\mathrm{r}}$ for the rupture that propagates or spreads starting from $\xi_{1}=0$ to $\xi_{1}=L$. In other words, the slip at the surface element $\boldsymbol{\xi}$ is activated with a delay given by $\tau(\boldsymbol{\xi})=\xi_{1} / c_{\mathrm{r}}$. The total far-field displacement $d$ due to the entire rupture is the sum of contributions from different surface elements:

$$
d(\mathbf{x}, t ; \Xi)=\int_{\Xi} h(\boldsymbol{\xi}) g(\mathbf{x}, t-\tau(\boldsymbol{\xi}) ; \boldsymbol{\xi}) \mathrm{d} \Xi
$$

the contributions being respectively delayed according to $\tau(\boldsymbol{\xi})$. We now assume that the dominant seismic wavelength $\lambda$ that is under consideration significantly exceeds the width $H$ of the fault, such that $g(\mathbf{x}, t ; \boldsymbol{\xi})$ will be in phase $\forall \xi_{2}$. Accordingly, we can rewrite the above equation using another scalar function $w$ as:

$$
d(\mathbf{x}, t)=\int_{0}^{L} w\left(\xi_{1}\right) g\left(\mathbf{x}, t-\frac{\xi_{1}}{c_{\mathrm{r}}} ; \xi_{1}\right) \mathrm{d} \xi_{1}
$$

In order to limit the dependency of $g$ on the length coordinate $\xi_{1}$ to an overall translation in time in eq. A.2, we make the so-called Fraunhofer approximation, which only makes an allowance for the far-field phase correction (travel-time difference) between 0 and $\xi_{1}$. For the part of the wavefield associated with waves having speed $c$ in the source region, we have

$$
g\left(\mathbf{x}, t ; \xi_{1}\right) \approx g\left(\mathbf{x}, t-\frac{\xi_{1} \cos \psi}{c} ; 0\right)
$$

where $\psi$ is the direction, relative to the rupture propagation, in which the waves depart from $\Xi$. Aki and Richards (2002) showed that this is a valid first-order approximation in a region, where the receivers are located at large distances

$$
\left|\mathbf{x}-\boldsymbol{\xi}_{0}\right| \gg \frac{2 L^{2}}{\lambda}
$$

Now, combining eqs. A.2 and A.3 and dropping the redundant argument 0 of $g$, we get:

$$
d(\mathbf{x}, t)=\int_{0}^{L} w\left(\xi_{1}\right) g\left(\mathbf{x}, t-\frac{\xi_{1} \gamma}{c_{\mathrm{r}}}\right) \mathrm{d} \xi_{1}, \quad \text { where } \quad \gamma=1-\frac{c_{\mathrm{r}} \cos \psi}{c}
$$

could be positive, negative or zero. For $\gamma \neq 0$, we now substitute $k=\xi_{1} \gamma / c_{\mathrm{r}}$ that belongs to a time interval 
$\mathbb{T}=\left\{t \in \mathbb{R} \mid 0<t c_{\mathrm{r}} / \gamma<L\right\}$ of length $|\gamma| L / c_{\mathrm{r}}$, to obtain

$$
d(\mathbf{x}, t)=\int_{-\infty}^{\infty} s(k ; \psi) g(\mathbf{x}, t-k) \mathrm{d} k,
$$

where the rupture manifests itself in the recorded time as a function commonly known as the apparent source time function (ASTF):

$$
\begin{aligned}
& s(t ; \psi)= \begin{cases}\frac{c_{\mathrm{r}}}{|\gamma|} w\left(\frac{t c_{\mathrm{r}}}{\gamma}\right) & \text { when } \gamma \neq 0 \& t \in \mathbb{T}, \\
0 & \text { otherwise }\end{cases} \\
& \underset{\gamma \rightarrow 0}{\longrightarrow} \delta(t) \int_{0}^{L} w(x) d x
\end{aligned}
$$

(a corollary of e.g., Stein and Weiss, 2016, Theorem 1.18). Finally, we time-discretize and rewrite eq. A.6 as a temporal convolution

$$
u *_{t} v=\sum_{k=-\infty}^{\infty} u(k) v(t-k)
$$

between the ASTF and the Green's function $g$ to obtain eq. 1. For finite signals, (A.7) becomes

$$
\left(u\left(T_{1}\right), u\left(T_{1}+1\right), \ldots, u\left(T_{2}\right)\right) *_{t}\left(v\left(T_{3}\right), v\left(T_{3}+1\right), \ldots, v\left(T_{4}\right)\right)=\sum_{k=\max \left(T_{1}, t-T_{4}\right)}^{\min \left(T_{2}, t-T_{3}\right)} u(k) v(t-k) .
$$

\section{Appendix B More Complex Earthquakes}

We analyzed a wide variety of earthquakes in our research, other than those discussed in this article. Most of them were complex, in the sense that it was difficult to interpret the extracted source spectrum directly via the redshift attribute. Therefore, additional spectral attributes have to be defined when continuing this research. Here, we present the source spectra of two slightly complex events, listed in Table B.1. The locations of these events and their corresponding stations are plotted in Figs. B.1a and B.1d, respectively.

We first present the FBD analysis for the July 5 mainshock USGS (2019b) in the Ridgecrest sequence. Compared to its foreshock, presented in the main text, the estimated auto-correlated source pulse $\hat{s}_{\mathrm{a}}$ in Fig. B.1b is complex. However, there is a minor indication that a dominant rupture mode is propagating towards the NW direction — note the longer source-pulse duration around $160^{\circ}$ azimuth. Nevertheless, its corresponding spectral-energy vs azimuth plot in Fig. B.1c was too complicated to interpret as a unilateral 
Table B.1: List of earthquakes, which are analyzed in the appendices, with two possible moment-tensor solutions. Courtesy: GEOFON Earthquake Information Service.

\begin{tabular}{lrrrccc}
\hline Name, Date, Mw & Latitude & Longitude & Depth & Strike $\left(^{\circ}\right)$ & Dip $\left(^{\circ}\right)$ & Rake $\left(^{\circ}\right)$ \\
\hline California, 2019-07-05, 7.1 & $35.76^{\circ} \mathrm{N}$ & $117.57^{\circ} \mathrm{W}$ & $14 \mathrm{~km}$ & 140,233 & 76,78 & 167,14 \\
Loyalty, 2017-10-31, 6.7 & $21.64^{\circ} \mathrm{S}$ & $169.21^{\circ} \mathrm{E}$ & $11 \mathrm{~km}$ & 154,321 & 76,14 & 93,77 \\
Tohoku, 2011-03-11, 9.0 & $38.3^{\circ} \mathrm{N}$ & $142.37^{\circ} \mathrm{E}$ & $15 \mathrm{~km}$ & 197,24 & 14,76 & 84,92 \\
Sumatra, 2012-04-11, 8.7 & $2.327^{\circ} \mathrm{N}$ & $93.063^{\circ} \mathrm{E}$ & $24 \mathrm{~km}$ & 289,20 & 83,85 & 175,7 \\
Kaikoura, 2016-11-13, 7.9 & $42.73^{\circ} \mathrm{S}$ & $173.054^{\circ} \mathrm{E}$ & $22 \mathrm{~km}$ & 225,342 & 28,77 & 150,66
\end{tabular}

propagation.

Similarly, the FBD analysis of a $\mathrm{Mw}=6.7$ earthquake to the Southeast of Loyalty Islands is presented in Figs. B.1d, B.1e and B.1f. The apparent source-time function estimated using the SCARDEC method (Vallée and Douet, 2016) indicated a duration of silence of about $5 \mathrm{~s}$ during the earthquake. This is consistent with the FBD result in Fig. B.1e, where $\hat{s}_{\mathrm{a}}$ exhibits a silence during the rupturing for about the same duration; the $\mathrm{P}$ waves from this earthquake are further analyzed in the next appendix. As a result, we conclude that the earthquake didn`t consist of a single rupture propagation with a constant velocity.

\section{Appendix C FBD of P-phases Using the Fresnel Approximation}

Similar to the factorization of surface waves, which was presented in section 4.3, focused blind deconvolution (FBD) can also factorize the $\mathrm{P}$ phases into respective source and path effects. In this case, in addition to the amplitude spectrum, the phase spectrum of the apparent source time functions can be estimated via focused phase retrieval (FPR, Bharadwaj et al., 2019). FPR assumes that the path effects are front-loaded, which is acceptable for the early $\mathrm{P}$ phases. The front-loaded assumption is impractical in the case of surface waves; therefore, the results in this paper were limited to the analysis of the source amplitude spectra. In this appendix, we present the FBD of the early P phases, and compare the results to those in the SCARDEC database (Vallée et al., 2011). Towards that end, we conveniently make the Fresnel approximation, where the earthquake source is modeled as a point source at epicentral distance $\gtrsim 10^{\circ}$. Vertical-component teleseismic records at Global Seismographic Network (GSN) stations, independent of the azimuth, are windowed using a rectangular function (width $\approx 500 \mathrm{~s}$ ) centered around the $\mathrm{P}$ arrival. The earthquakes and station locations of the windowed records that are input to FBD are plotted in Figure C.1. The earthquakes along with the moment-tensor solutions are listed in Table B.1. The FBD estimated source time functions $\hat{s}$ are presented in black in Figure C.1. Notice that the source-time durations and the rise times i.e., the time taken by the slip to reach its maximum value, correlate well with the SCARDEC results (in blue). Also, similar to Appendix B, the $\hat{s}$ of Loyalty earthquake again exhibits the silence duration. We conclude this appendix by stating that the results of FBD agree well with the established observations. 
(a)

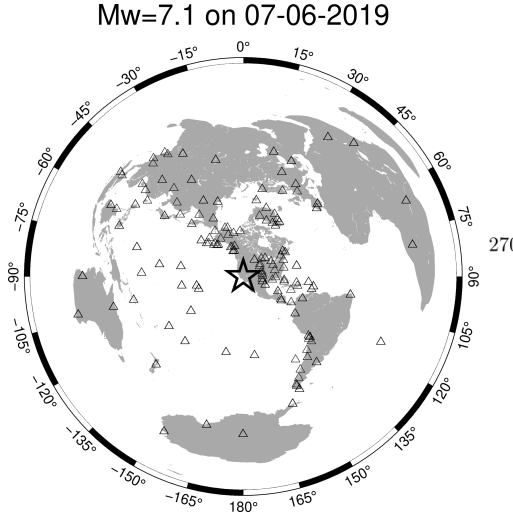

(d)

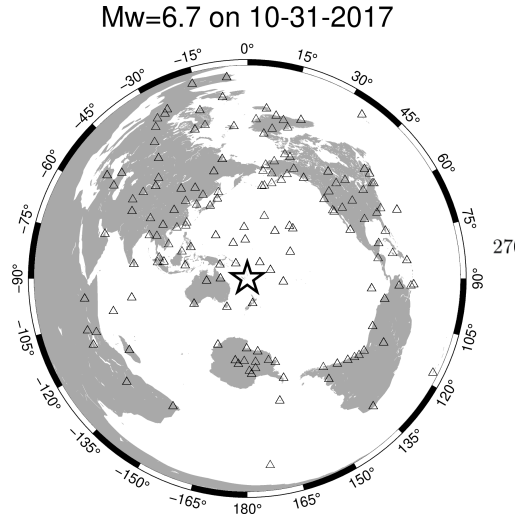

(b)

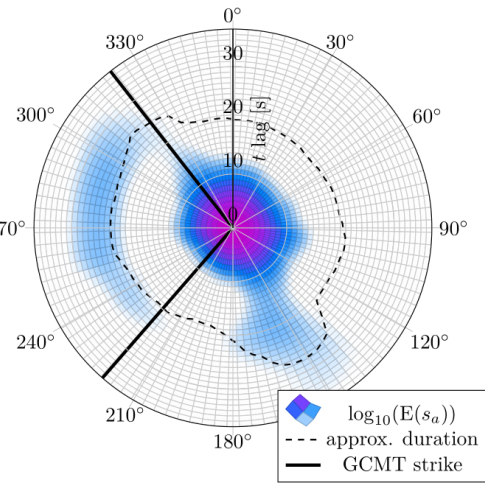

(e)

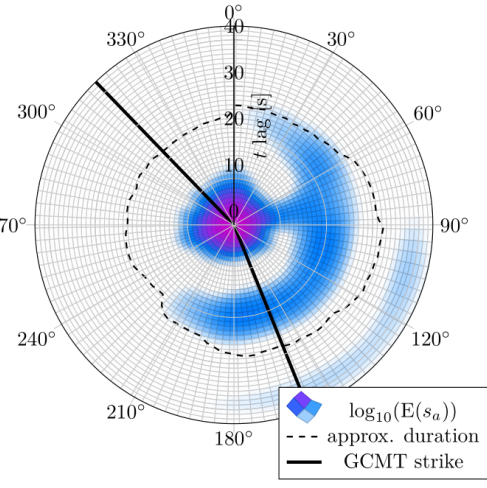

(c)
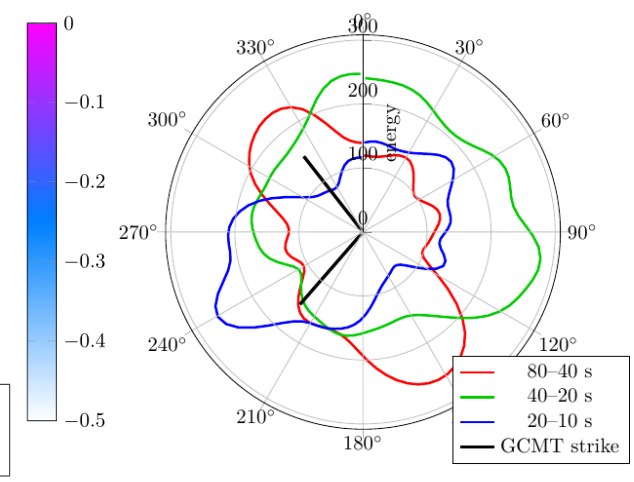

(f)

Figure B.1: As in Fig. 3, except that the FBD results indicate complex rupturing. Redshift due to a unilateral rupture propagation cannot be identified during the analysis of these earthquakes, which are listed in the Table B.1. 
a) 11-Mar-2011 Tohoku $\mathrm{Mw}=9.0$
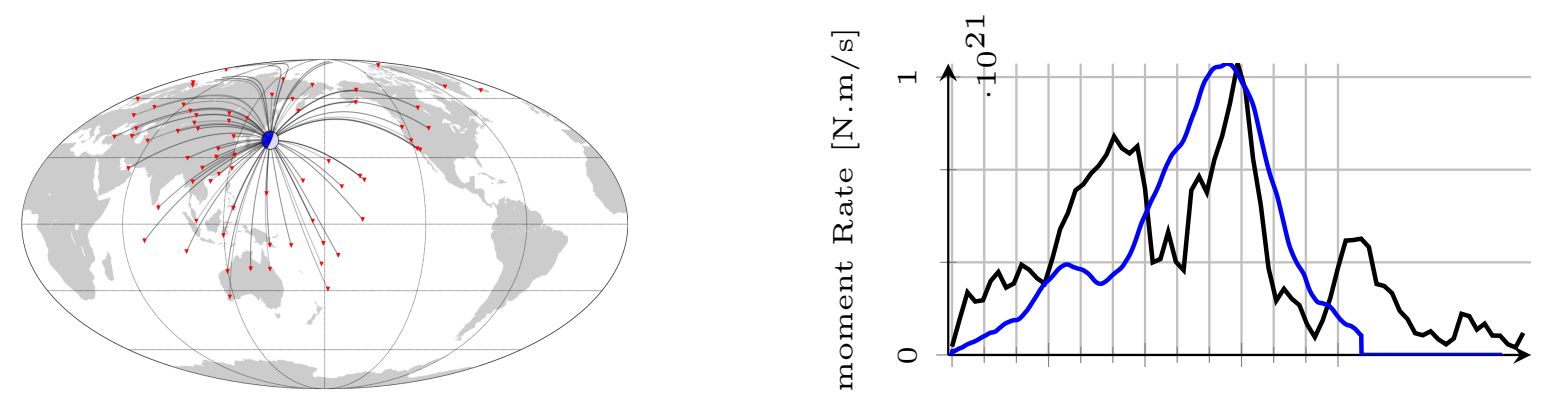

b) 11-Apr-2012 Sumatra $\mathrm{Mw}=8.7$
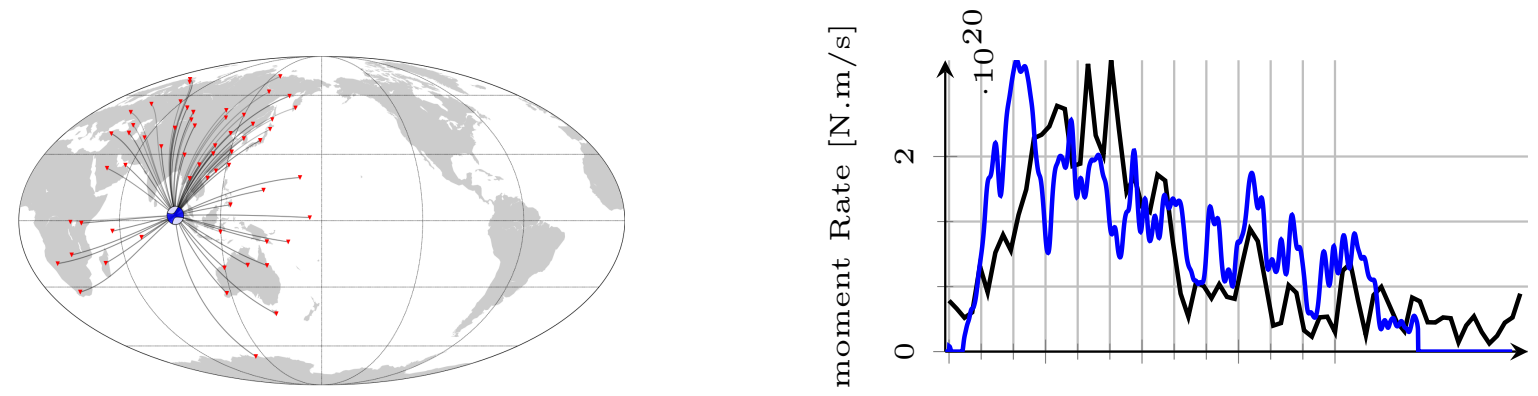

c) 13-Nov-2016 Kaikoura $\mathrm{Mw}=7.9$
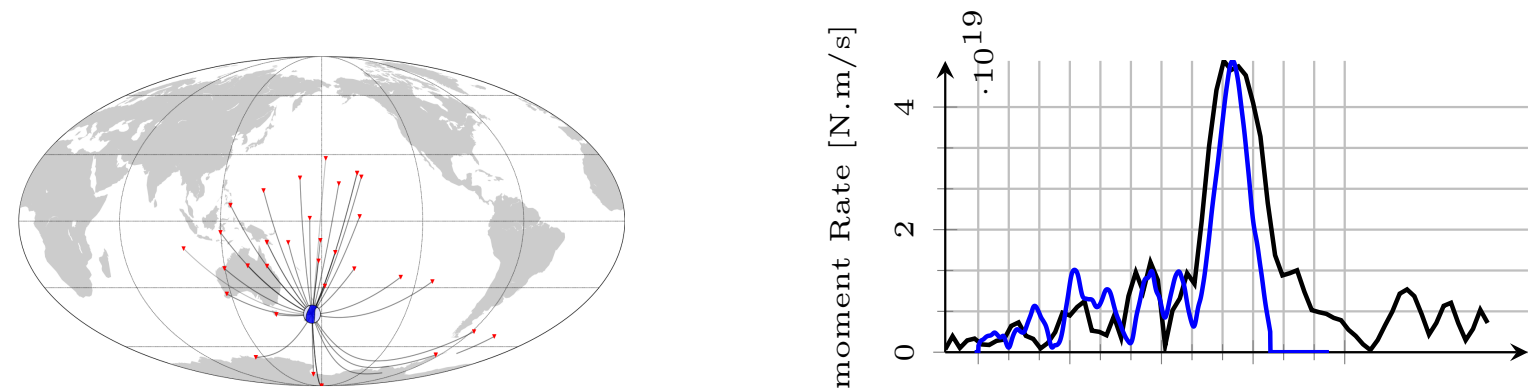

d) 31 -Oct-2017 Loyalty $\mathrm{Mw}=6.5$
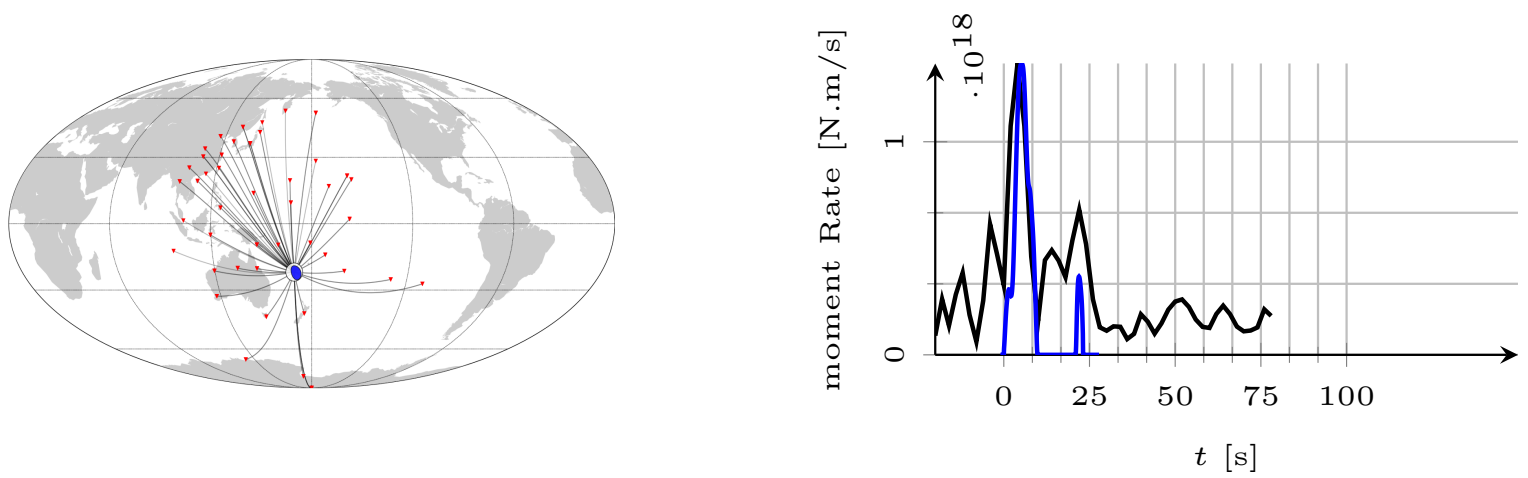

Figure C.1: FBD estimated P-wave apparent-source-time functions for five different earthquakes with magnitudes $6.5<\mathrm{Mw}<9.0$ are plotted in black. For comparison, the ASTFs estimated using the SCARDEC method (Vallée et al., 2011) are plotted in blue. GSN stations are plotted in red. 


\section{${ }_{539}$ Appendix D Software: FocusedBlindDecon.jl}

540 We have made documented software available to perform focused blind deconvolution through a Julia pack-

541 age: https://github.com/pawbz/FocusedBlindDecon.jl. 